\title{
The Impact of the COVID-19 Pandemic on Ophthalmology Resident Perceptions of Clinical Experience, Surgical Training, and Personal Life
}

\author{
Marissa K. Shoji, MD ${ }^{1}$ Michael J. Venincasa, MD ${ }^{1}$ \\ ${ }^{1}$ Department of Ophthalmology, Bascom Palmer Eye Institute, \\ University of Miami Miller School of Medicine, Miami, Florida \\ 2 Department of Vitreoretinal Surgery, Bascom Palmer Eye Institute, \\ University of Miami Miller School of Medicine, Miami, Florida
}

\author{
Jayanth Sridhar, $\mathrm{MD}^{1,2}$
}

J Acad Ophthalmol 2021;13:e288-e297.

\begin{abstract}
Address for correspondence Jayanth Sridhar, MD, Bascom Palmer Eye Institute, University of Miami Miller School of Medicine, 900 N.W. 17th Street, Miami, FL 33136 (e-mail: Jsridhar1@med.miami.edu).
\end{abstract}

received

February 2, 2021 accepted after revision July 30, 2021
Objective The coronavirus disease 2019 (COVID-19) pandemic has affected multiple areas of health care, including residency training programs. Elucidating the effect of the COVID-19 pandemic on resident clinical experience, surgical training, and wellness is essential to identify areas in which programs can provide additional educational and personal resources to trainees. This study aims to evaluate the experiences of ophthalmology residents during the pandemic.

Design The design is a cross-sectional, nonvalidated survey study. The survey was administered online with data collection from August 22 to August 31, 2020.

Participants Applicants to the Bascom Palmer Eye Institute ophthalmology residency program during the 2016 to 2019 application cycles were invited to complete the survey to encompass trainees currently in ophthalmology residency during the COVID19 pandemic. Applicants who were not training at an ophthalmology residency program during the pandemic were excluded.

Methods This study involved eliciting feedback from ophthalmology residents on the perceived impact of COVID-19 on their residency experiences through survey questions.

Main Outcome Measures Perceived didactic, clinical, surgical, and overall experiences of residents during the COVID-19 pandemic, effect on cataract and noncataract surgical case volume, and perceived effects on resident personal life.

Results Responses were obtained from 357 (22.8\%) individuals, 193 of which met inclusion criteria (59.1\% male, $54.9 \%$ aged $30-34)$. Most participants reported overall worsening of their ophthalmology training experience due to COVID-19 (75.1\%), with worsening of clinical training reported by $72.5 \%$ of participants and worsening of surgical training reported by $89.1 \%$ of participants. There were no significant differences in the perception of the impact of COVID-19 on overall training experience, clinical training experience, or surgical training experience among geographic regions $(p=0.43, p=0.23, p=0.27$, respectively). A higher percentage of post-graduate year 3
DOI https://doi.org/ $10.1055 / \mathrm{s}-0041-1740314$ ISSN 2475-4757.

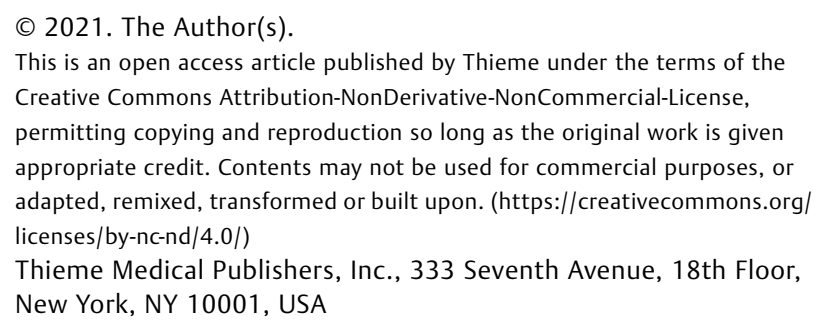
appropriate credit. Contents may not be used for commercial purposes, or adapted, remixed, transformed or built upon. (https://creativecommons.org/ licenses/by-nc-nd/4.0/) Thieme Medical Publishers, Inc., 333 Seventh Avenue, 18th Floor, New York, NY 10001, USA 
(PGY3) and PGY4 trainees reported worsened clinical $(p=0.003)$ or surgical $(p=0.03)$ experience compared with $\mathrm{PGY} 2$ trainees. Participants also reported impact on personal life including time spent away from family (52.9\%), worsened friendships with co-residents (29.5\%), forced changes in living situation (15.0\%), and increased expenses (13.5\%).

Conclusion The COVID-19 pandemic has substantially impacted clinical and surgical experience of ophthalmology residents, who also report personal stressors due to the pandemic. Identifying alterations in the ophthalmology residency experience is essential to provide additional resources to support ophthalmology trainees professionally and personally during this time.

Since 2020 , there has been characterized by apprehension and challenges due to the global outbreak of the severe acute respiratory syndrome coronavirus 2 (coronavirus disease 2019 [COVID-19]) and subsequent pandemic, with more than 25.1 million COVID-19 cases worldwide and more than 6 million cases in the United States (U.S.) by the end of August 2020. ${ }^{1}$ This pandemic has led to significant changes in U.S. ophthalmology residency clinical training, including redeployment of ophthalmology residents to work on the frontlines in non-ophthalmology fields, implementation of practices to protect asymptomatic patients and providers, and adoption of social distancing methods for teaching and patient care such as virtual didactics and teleophthalmology. ${ }^{2-4}$ Moreover, clinical and surgical volumes have been impacted, as many hospitals throughout the United States responded to the pandemic by reducing nonessential clinical visits, procedures, and surgeries. ${ }^{4,5}$ While this anecdotally has significantly affected the ophthalmology clinical and surgical experience in residency programs, no studies have yet assessed the experience of ophthalmology residents in the United States to better quantify these changes.

Additionally, COVID-19 has also likely impacted residents not only due to professional concerns, including maintaining education, changes in essential examination scheduling, and employment worries, but also due to increased personal stressors, including potential redeployment and its associated challenges, financial and professional concerns, risk of infection during patient care, or loved ones suffering from COVID-19 complications. ${ }^{2,6,7}$ Surgical residency training has been demonstrated to be associated with burnout in the absence of a pandemic ${ }^{8-10}$; therefore identification of personal stressors during the pandemic is critical to avoid exacerbation of burnout, which is important due to moral, ethical, and professional implications including maintaining patient safety and quality of care. ${ }^{11-16}$

Assessing the impact of the pandemic on ophthalmology residents is essential; outbreaks of the new variants of the virus threaten to result in persistence of the pandemic, and the effects of the pandemic will likely endure throughout the eventual recovery process and ultimate establishment of a "new normal." Moreover, insights obtained from this pandemic may be applicable to potential similar situations in the future. Therefore, this study aims to evaluate the effect of
COVID-19 on the perceived experience of ophthalmology residents throughout the United States.

\section{Methods}

This study was a cross-sectional, nonvalidated survey study conducted at Bascom Palmer Eye Institute. Institutional Review Board (IRB) approval was obtained, and the study adhered to the Declarations of Helsinki. An online, anonymous survey was distributed to ophthalmology trainees who previously applied for residency at Bascom Palmer during the 2016 to 2017,2017 to 2018, and 2018 to 2019 application cycles (those in their post-graduate year [PGY] 1-3 in the 2019 to 2020 academic year), and for fellowship at Bascom Palmer during the 2019 to 2020 application cycle (those in their PGY4 in 2019-2020). Respondents who did not successfully match to any ophthalmology residency, as well as those who were not part of an "integrated" PGY-1 year that included formal ophthalmology training, were excluded. This allowed for the evaluation of the experience of trainees participating in ophthalmology residency training, regardless of whether they matched at Bascom Palmer or were participating in other residency programs throughout the United States, in March 2020, during the first U.S. COVID-19 surge, through August 2020. Data were collected from August 22, 2020 to August $31^{\text {st }}, 2020$. Survey creation, distribution, and data collection were similar to a prior study conducted by our group. ${ }^{17}$ Participation was voluntary, and no compensation was provided. Informed consent was obtained, and participants were allowed to opt out at any time during the survey.

Data collected included participant demographics, including age, sex, and location of residency program. Location was categorized into regions as per the U.S. Census guidelines including Northeast, Southern, Midwest, and Western U.S. Additional collected data included resident perception of didactic, clinical, surgical, and overall experience and volume at the time in which residents felt their training was most affected and at the time of survey administration, number of expected and actual cataract and noncataract surgeries performed between March $1^{\text {st }}, 2020$ and the date of survey administration, research output, redeployment to clinical responsibilities outside of 
ophthalmology training, overall satisfaction with the residency program, and nonvalidated assessment of the effect on resident personal life.

Association between categorical variables was assessed using Chi-squared test for categorical variables or Student's ttest for quantitative variables. $p<0.05$ was considered statistically significant.

\section{Results}

The survey invitation was sent to 1,559 separate email addresses. Fifty-nine messages were returned due to inactive email accounts. The survey invitation email was opened by 449 (28.8\%) of those invited, and responses were initiated by 357 (22.8\%), with 69 incomplete responses. For the remaining 288 complete responses, 24 respondents were excluded due to failure to match into an ophthalmology residency, 60 were excluded due to completion of a PGY-1 year that was not classified as an integrated ophthalmology residency during the specified time frame, and 11 were excluded due to being a nonresident during the 2019 to 2020 academic year.

Complete responses were received from 193 (12.4\%) respondents and were included in the study. Most respondents were males $(N=114 ; 59.1 \%)$ between 30 and 34 years of age $(N=106 ; 54.9 \%)$ located in the Northeast $(N=69$; $35.8 \%)$ or Southern United States $(N=55 ; 28.5 \%$, - Table 1$)$. The geographic distribution of respondents was statistically similar to geographic distribution of all ophthalmology residency positions $\left(X^{2}=2.21 ; p=0.53\right)$.

Most respondents felt that COVID-19 "worsened" their overall training experience $(N=145 ; 75.1 \%)$, with no significant differences among geographic locations $(\boldsymbol{p}=0.30$, - Table 2). Regarding the effect of COVID-19 on various aspects of training, the most common response was in-office/clinical $(N=103 ; 53.4 \%)$ and surgical training $(N=87 ; 45.1 \%)$ was "slightly worsened," but many $(N=85$; $44.0 \%$ ) felt surgical training was "significantly worsened" and didactic training "neither improved nor worsened" $(N=57$; $29.5 \%)$ or "slightly improved" ( $N=51 ; 26.4 \%$ ). These perceptions did not differ among locations ( $p>0.05$ for all) but did significantly differ for overall effect on in-office/clinical

Table 1 Respondent demographics

\begin{tabular}{|l|l|}
\hline Gender $(N=193)$ & \\
\hline Male & $114(59.1 \%)$ \\
\hline Female & $77(39.9 \%)$ \\
\hline Prefer not to say & $2(1.9 \%)$ \\
\hline Age (years, $N=193)$ & \\
\hline$<25$ & $1(0.5 \%)$ \\
\hline $25-29$ & $69(35.8 \%)$ \\
\hline $30-34$ & $106(54.9 \%)$ \\
\hline $35-39$ & $14(7.3 \%)$ \\
\hline$>39$ & $3(1.6 \%)$ \\
\hline
\end{tabular}

( $p=0.0003)$ and surgical $(p=0.03)$ training based on PGY year, with a higher percentage of PGY3 and PGY4 participants reporting slightly or significantly worse effects on their experiences due to COVID-19 (-Table 3). Respondents reported completing an average of $44.6 \%$ of their expected number of cataract surgeries $(\mathrm{SD}=80.9 \% \%$; range $=0$ $1,000 \%$ ) and $49.6 \%$ of their expected number of noncataract surgeries ( $S D=31.9 \%$; range $=0-167 \%)$; these differences in expected and actual cataract and noncataract surgeries did not differ among regions ( $p=0.74, p=0.74$, respectively). At the time of survey completion, most participants felt that in-office/clinical volume was at 75 to $99 \%(N=96 ; 49.7 \%)$, surgical volume at 50 to $74 \%(N=66 ; 34.2 \%)$, and research volume at 100 to $124 \%(54 ; 28.0 \%)$ compared with preCOVID-19 volumes.

Most respondents $(N=159 ; 82.4 \%)$ were informed that they may be required to "redeploy" into work in a nonophthalmology specialty with 33 trainees (17.1\%) reporting actual redeployment. While the possibility of redeployment did not differ based on location $(p=0.56)$, the likelihood of actual deployment did differ $(p<0.0001)$ with the highest chance occurring in the Northeast $(N=26 ; 37.7 \%)$ and the lowest in the Southern United States residencies $(N=2$; 3.6\%). Most residents were either "satisfied" or "very satisfied" with residency program policies regarding COVID-19related changes $(N=122 ; 63.2 \%)$, communication $(N=133$; $68.9 \%)$, personal protective equipment policies $(121 ; 62.7 \%)$, and COVID-19 testing of patients ( $N=116 ; 60.1 \%)$, but less so regarding COVID-19 testing of residents $(N=82$; 42.5\%, - Table 4).

The effect of COVID-19 on residents' personal lives varied but was mostly negative, including time spent away from family due to quarantine $(N=104 ; 54.5 \%)$, increased expenses $(N=26 ; 13.6 \%)$, and worsened relationships with co-residents $(N=57 ; 29.8 \%)$ (-Table 5); write-in responses for this question highlighted difficulties regarding childcare, as well as concerns for dichotomy in surgical volume based on whether surgery-heavy rotations were scheduled before or during the pandemic.

\section{Discussion}

Residency training has been substantially affected by the COVID-19 pandemic throughout the United States and internationally. This is the first study to quantitatively assess the perception of the impact of this pandemic on U.S. ophthalmology resident experiences as well as contribution of the pandemic to personal stressors.

Overall, most trainees reported a negative perception of their clinical and surgical experience during this time ("slightly worsened" or "significantly worsened"). The reduced number of cataract and non-cataract cases, which likely occurred due to cancellation of nonessential surgeries, substantiate this negative perception of surgical experience. Currently, graduation from U.S. ophthalmology residency requires completion of 86 cataract surgeries. ${ }^{18}$ While the present survey is unable to identify whether residents continued to meet required case numbers, respondents 


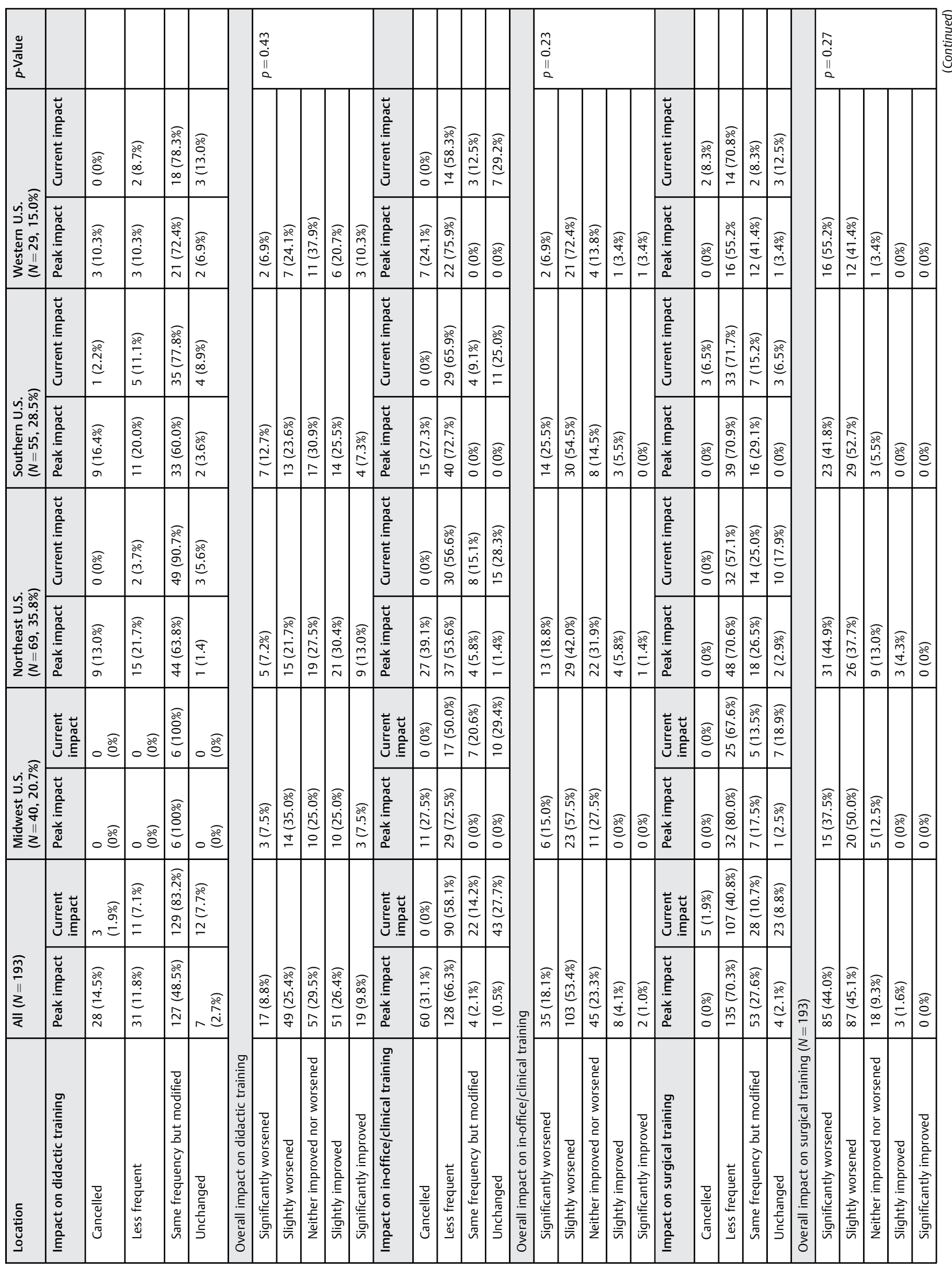




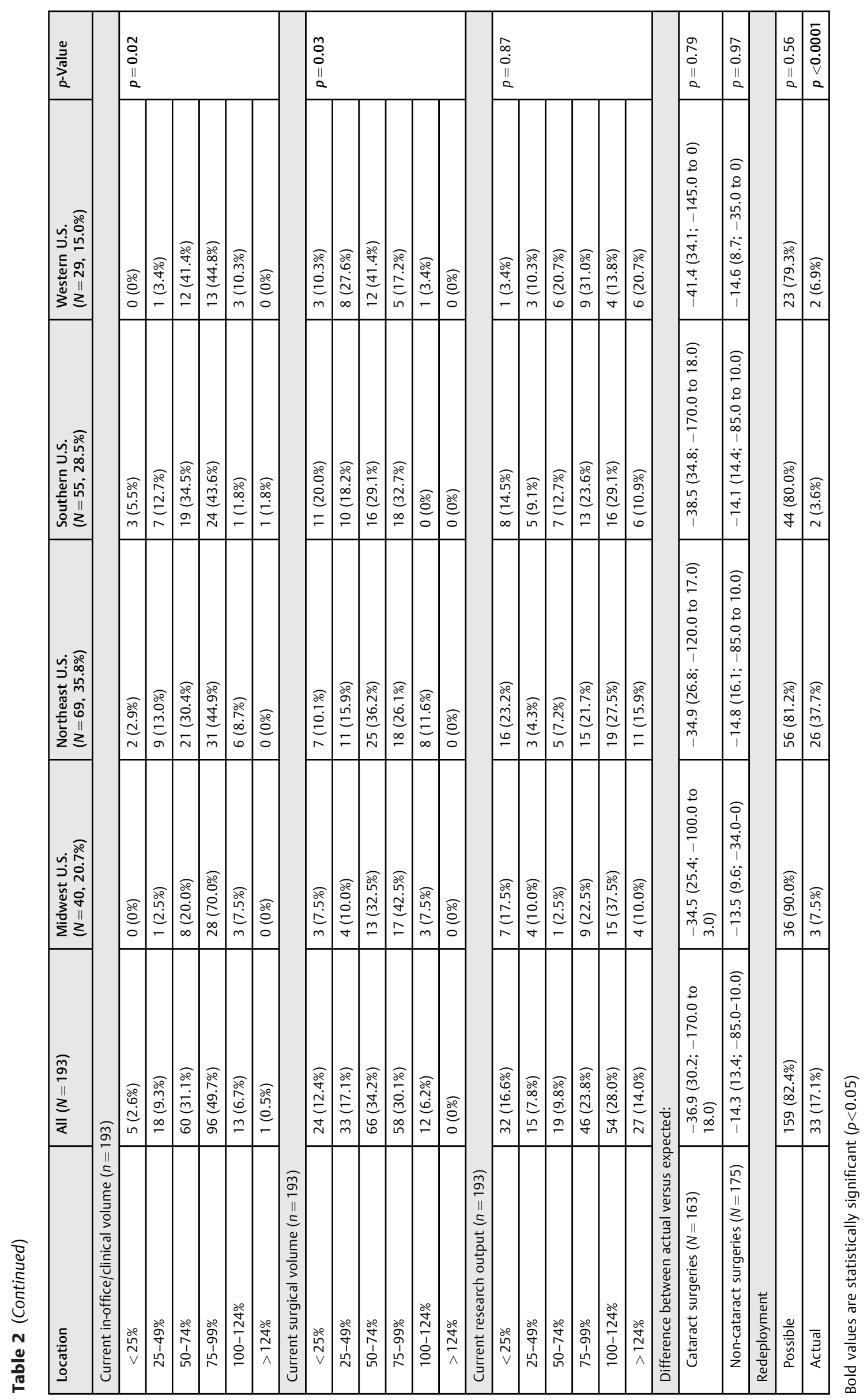




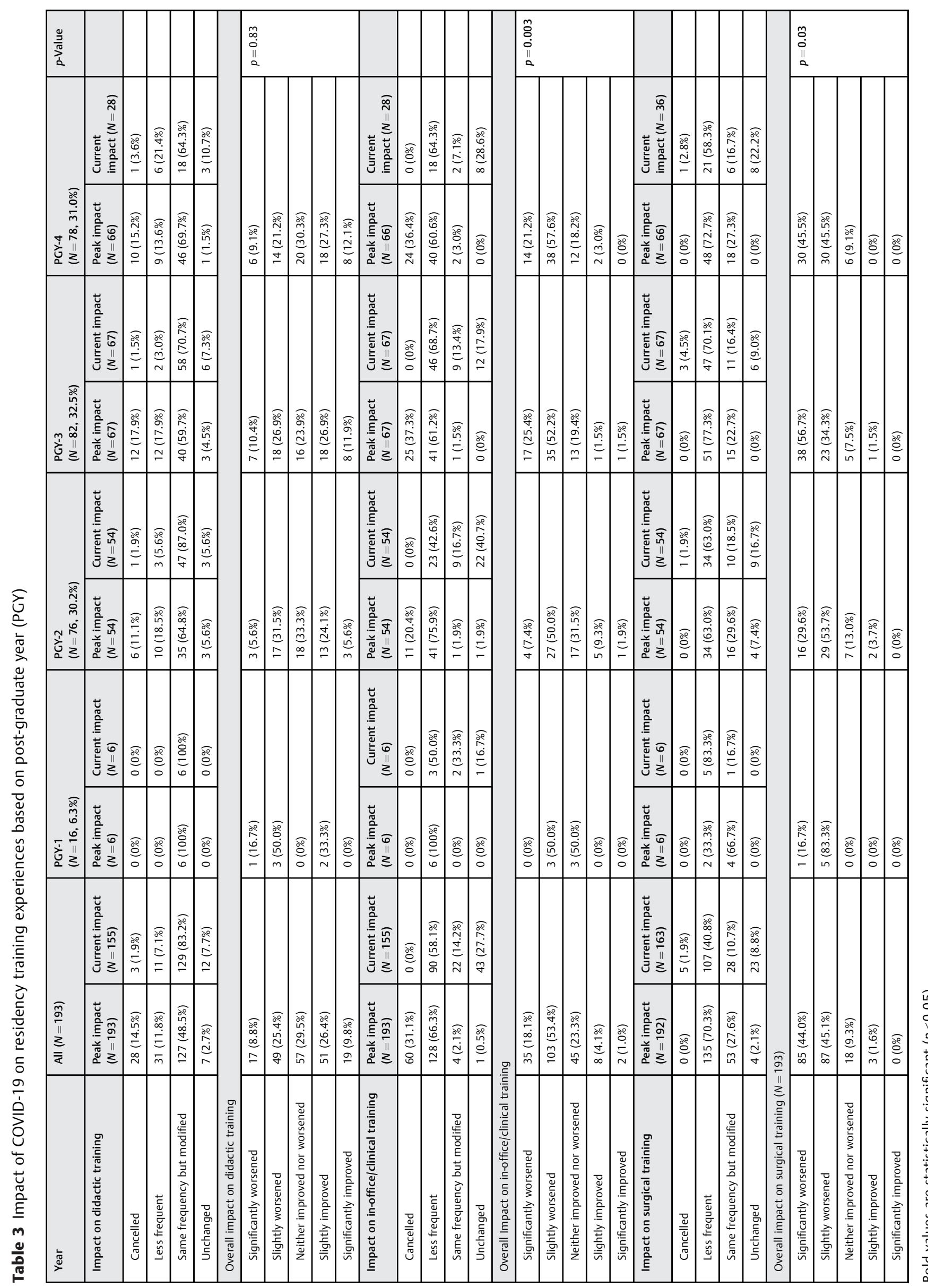


e294 Impact of the COVID-19 Pandemic on Ophthalmology Residents Shoji et al.

Table 4 Resident satisfaction with program COVID-19 policies

\begin{tabular}{|l|l|l|l|l|l|}
\hline Satisfaction with $(N=193):$ & Changes & Communication & PPE policies & Patient testing & Resident testing \\
\hline Very dissatisfied & $8(4.2 \%)$ & $3(1.6 \%)$ & $9(4.7 \%)$ & $9(4.7 \%)$ & $20(10.4 \%)$ \\
\hline Dissatisfied & $25(13.0 \%)$ & $24(12.4 \%)$ & $27(14.0 \%)$ & $22(11.4 \%)$ & $41(21.2 \%)$ \\
\hline Neither satisfied nor dissatisfied & $38(19.7 \%)$ & $33(17.1 \%)$ & $36(18.7 \%)$ & $46(23.8 \%)$ & $50(25.9 \%)$ \\
\hline Satisfied & $87(45.1 \%)$ & $74(38.3 \%)$ & $72(37.3 \%)$ & $78(40.4 \%)$ & $53(27.5 \%)$ \\
\hline Very satisfied & $35(18.1 \%)$ & $59(30.6 \% \%)$ & $49(25.4 \%)$ & $38(19.7 \%)$ & $29(15.0 \%)$ \\
\hline
\end{tabular}

Table 5 Effect of COVID-19 on respondent personal life

\begin{tabular}{|l|l|}
\hline Respondent $(\boldsymbol{N}=\mathbf{1 9 3})$ : & \\
\hline Time spent away from family & $104(53.9 \%)$ \\
\hline Increased time to spend with family & $79(40.9 \%)$ \\
\hline “Forced" changes in living situation & $29(15.0 \%)$ \\
\hline Increased expenses & $26(13.5 \%)$ \\
\hline Improved friendships with co-residents & $38(19.7 \%)$ \\
\hline Worsened friendships with co-residents & $57(29.5 \%)$ \\
\hline
\end{tabular}

reported an average of only $44.6 \%$ of their expected number of cataract cases between March 2020 and the time of survey completion; one can presume that surgical training was adversely affected, especially for residents who scheduled surgery-heavy rotations during these affected months and those participating in programs in which surgical training occurs primarily during the PGY4 year. While in-office/clinical volume was perceived as returning to 75 to $99 \%$ at the time of the survey compared with prepandemic, $63.7 \%$ of respondents perceived surgical volume as less than $75 \%$ of pre-pandemic volumes, with only $12.2 \%$ of respondents reporting return of volume to 100 to $124 \%$ of pre-pandemic volume. This suggests that the surgical experience is particularly susceptible to the impact of the pandemic without appropriate compensatory return of volume; therefore, one important area of focus for training programs is augmentation of the resident surgical experience.

Interestingly, the perceived impact of COVID-19 pandemic on didactic, clinical, and surgical training did not vary among regions despite the temporal differences in the COVID-19 case volume surge by region, as the first COVID19 surge occurred sooner in the Northeast compared with the Midwest, South, and Western regions. ${ }^{19}$ This may be due to the design of the study, which allowed participants to define what they considered as the peak. Additionally, patients and hospitals may have modified their behavior, including cancellation of elective surgeries or fewer patients presenting for nonessential visits, due to concern about the pandemic even if they were located in regions with relatively fewer COVID-19 cases. Regardless of region, most ophthalmology trainees nationwide deemed their overall clinical and surgical experience to have been negatively impacted by the pandemic suggesting that trainees in programs in all locations, even those with relatively fewer COVID-19 cases, may require dedicated efforts to enhance their residency experience.

Other studies have investigated the impact of the pandemic on other subspecialties; notably, there have been reports of the impact of the pandemic on urology, otolaryngology, and plastic surgery training, which may yield useful insights as these are relevant surgical subspecialties with numerous nonemergent procedures similar to ophthalmology. ${ }^{20-24}$ Multiple other fields have also reported effects on the residency experience, including reduction in training and surgical volume, anxiety about professional future, and indelible compromise on their training. ${ }^{20-22,25-27}$ These impacts are reported among different specialties both internationally ${ }^{25,28-30}$ and within the United States ${ }^{20,26,27}$ despite international differences in residency structure, suggesting that insights regarding the effect of the pandemic on the training experience may transcend geographical borders and specialties.

International studies assessing the effect of the pandemic on ophthalmologists and ophthalmology trainees have reported significant negative impact of the pandemic on the overall experience with reductions in clinical and surgical volume. ${ }^{31-34}$ These findings parallel the results from our study including the effect on clinical and surgical training. Within the United States, ophthalmology program directors in New York City (NYC) offered perspective on modifications to residency programs, including telemedicine, virtual lectures, and residency support meetings. ${ }^{4}$ Bakshi et al similarly provides commentary on suggested modifications to the residency experience without directly sharing the trainee perspective. ${ }^{2}$ Other proposed modifications include surgical videos and simulation exercises such as a remote cornea wet laboratory and oculoplastics virtual curriculum, which may help address the decreased volume and worsened surgical experience reported by respondents in our study. ${ }^{35-37}$ Two publications involve firsthand accounts of ophthalmology resident experiences in Iowa and NYC, ${ }^{7,38}$ in which both redeployed and nondeployed residents discuss the influence of the pandemic on their residency experience. These perspectives echo many of the findings in our study, including clinical, surgical, and didactic modifications, changes in social support and loneliness experienced by trainees, and alterations in approach to patient care, with additional anxiety and stress resulting from fear of possible redeployment from ophthalmology to taking care of COVID-19 
patients and the enormous emotional, mental, and physical tolls associated with redeployment.

Our study also assesses the effect of the pandemic on personal lives of ophthalmology trainees. More respondents reported increased time spent away from family (53.9\%) compared with those reporting increased time to spend with family (40.9\%). Co-resident interpersonal relationships were also affected, with $29.5 \%$ reporting worsened friendships with co-residents compared with $19.7 \%$ reporting improved friendships during this time. This decreased time with family and co-residents is important to identify as loneliness may be associated with burnout despite reduced work hours. ${ }^{39}$ Studies have investigated the psychological impact of COVID-19 on mental health outcomes including depression, anxiety, and insomnia of practicing ophthalmologists and trainees; 32.6 to $50 \%$ of ophthalmologists reported symptoms of depression, $46.7 \%$ reported anxiety symptoms, and $15 \%$ reported insomnia. ${ }^{31,40,41}$ While the baseline mental health status of ophthalmologists prepandemic is unknown, possible stressors include pervasive uncertainty inherent in the pandemic, concern for patient, personal, and family risks of infection, loved ones suffering from complications of COVID-19 including hospitalization and death, stigmatization targeting health care professionals by mainstream media, and effects on career due to reduced patient volume. ${ }^{40}$ As the pandemic continues, it is imperative to consider implementing support for the emotional and mental health of ophthalmology trainees to facilitate trainee well-being and maintain quality of patient care. ${ }^{4}$

The pandemic has resulted in a multifaceted influence on the ophthalmology trainee experience, with short-term and long-term effects on clinical and surgical training as well as personal stressors that may lead to reduced well-being. It is essential to develop and implement strategies to address these factors to maximize the residency experience, as the impact of the pandemic may extend well into the future especially with the continual increase in COVID-19 cases.

Online didactics appear to be an effective method of addressing the challenges of teaching while maintaining appropriate social distance. ${ }^{31}$ Additional advantages of these online didactics include the possibility of recording lectures for future availability, ability to adjust lecture time and location convenient to the attendees and lecturer, and expansion of didactics to those who may not have had access previously. Prior studies reported that transition to online and virtual lectures and conferences was considered valuable. ${ }^{31}$ The implementation of these resources may result in the perceived lack of effect of the pandemic on the didactic experience reported by U.S. ophthalmology residency trainees in this study.

Augmentation of clinical experiences may include virtual clinics, telemedicine, and telephone consultations, which may lead to a shift toward routine use of virtual clinics and novel technology in the future. ${ }^{42,43}$ Despite the decrease in in-person volume, patients still present with vision and possible life-threatening pathology, with one study demonstrating that patients with conditions leading to vision changes, pain and photophobia, ocular malignancies, ocular trauma, and retinal pathology resulting in vision loss or requiring intravitreal injections continued to present for in-person evaluation during the COVID-19 pandemic. ${ }^{44} \mathrm{Ad}-$ ditionally, patients may present with pathology as a direct or indirect effect of COVID-19; ophthalmic manifestations of COVID-19 include follicular conjunctivitis, anterior uveitis, retinal changes including microhemorrhages and cotton wool spots, optic neuritis, and cranial nerve palsies, ${ }^{45}$ and additional cases reported include rhino-orbital mucormycosis in a patient with COVID-19, orbital emphysema due to intubation for COVID-19 pneumonia, and UV keratopathy due to increased consumer purchase and exposure to commercial UV light disinfectants during the pandemic. ${ }^{46-49}$ Thus, although methods to increase clinical volume should be implemented, the potential for high-quality patient encounters and experiences with interesting pathology persists despite decreased volume. Of note, although patients continued to present with emergent and urgent ophthalmic pathology, the pandemic may have resulted in the avoidance or delay in care for some individuals who may eventually present with more severe stages of their ophthalmic condition; further studies are required to evaluate this in detail.

The pandemic has especially affected resident surgical experience due to cancellation of elective cases and decreased volume. While not a direct substitute for operating, surgical videos may be an alternative to observation of cases that may develop surgical and nontechnical skills including decision-making and surgical planning. ${ }^{50}$ Interestingly, acquisition of both motor and cognitive skills may be achieved through observation. ${ }^{51}$ This suggests that recording and publishing surgical videos online may be useful to facilitate learning; moreover, this may promote an online forum for discussion of different techniques to collaboratively share knowledge and ideas beyond what may have occurred with a single in-person, unrecorded operating room observation. However, while observation and surgical videos are important, muscle memory is also essential to develop fine motor skills and techniques for ophthalmologic surgery. Simulation-based tools may be key to develop techniques to maintain and augment surgical skills; surgical simulation has been shown to positively impact ophthalmology resident surgical skills, including shorter phacoemulsification times, less phacoemulsification power, and lower complication rates. $^{52,53}$ Although data are equivocal regarding whether simulation-based training, including model simulation, is superior to standard surgical training, ${ }^{54,55}$ simulation-based training may allow for development of skills that may be transferable to the operating room after surgical volume returns to normal. ${ }^{56}$ of note, simulation-based training should not replace surgical training but rather may serve as a temporizing measure to maintain surgical skills in the setting of unavoidable transiently decreased volume.

While this study provides insight into the experience of ophthalmology residents during the pandemic, it has several limitations. Participants were included based on applications to the Bascom Palmer residency and fellowship programs, which may not capture all ophthalmology residents impacted by the pandemic. Since there are limited published 
demographics regarding the overall population of ophthalmology residents, it is difficult to compare our respondents to nonrespondents and to the population. However, the geographic distribution of respondents did not differ significantly from the geographic distribution of ophthalmology residency positions, suggesting that respondents may be representative, at least geographically, of the overall resident population. Recall bias and response bias are also inherent limitations of survey studies. Not all invited participants opened or completed the survey, which may be an additional source of participation bias, although $22.8 \%$ of those who opened the email with the survey invitation completed the survey. Our study attempts to provide both qualitative and quantitative data regarding perceived experiences in residency, which may lead to subjectivity. Our study also aimed to evaluate the resident experience at the perceived worst time of COVID and the present time, which may have flaws including geographic bias due to the variation in the time course of COVID-19 impact on the health care system within each region, although there was no statistically significant difference in perceived impact among different regions. Moreover, we included the perceived experience of PGY4 trainees at the time of the survey, which may involve their first year of practice in either fellowship or as an attending following graduation from residency and therefore may not reflect the true residency trainee experience.

COVID-19 has substantially impacted the clinical and surgical experience of ophthalmology residents throughout the United States. This impact is likely to persist as the pandemic continues and a "new normal" is ultimately established. It is important to identify gaps in training to create novel learning methods and creatively apply emerging technologies to augment the training experience and provide a new foundation for the didactic, clinical, and surgical experience that will endure the challenges of this pandemic. Moreover, insights from this pandemic may be applicable in optimizing resident experience and allocation of resources in potential additional surges of COVID-19 and future pandemics. Connections and collaboration are essential for enhancing the training experience as well as providing mutual support and solidarity among colleagues as we face the adversity of this time, serving patients and navigating this pandemic together.

\section{Funding}

None.

Conflict of interest

None declared.

\section{References}

1 WHO Coronavirus Disease (COVID-19) Dashboard. Accessed July 25, 2020. https://covid19.who.int

2 Bakshi SK, Ho AC, Chodosh J, Fung AT, Chan RVP, Ting DSW. Training in the year of the eye: the impact of the COVID-19 pandemic on ophthalmic education. Br J Ophthalmol 2020;104 (09):1181-1183
3 Chatziralli I, Ventura CV, Touhami S, et al; International Retina Collaborative. Transforming ophthalmic education into virtual learning during COVID-19 pandemic: a global perspective. Eye (Lond) 2021;35(05):1459-1466

4 Chen RWS, Abazari A, Dhar S, et al. Living with COVID-19: a perspective from New York Area Ophthalmology Residency Program Directors at the Epicenter of the Pandemic. Ophthalmology 2020;127(08):e47-e48

5 Wong TY, Bandello F. Academic Ophthalmology during and after the COVID-19 Pandemic. Ophthalmology 2020;127(08):e51-e52

6 Breazzano MP, Shen J, Abdelhakim AH, et al; New York City Residency Program Directors COVID-19 Research Group. New York City COVID-19 resident physician exposure during exponential phase of pandemic. J Clin Invest 2020;130(09):4726-4733

7 Rosenberg JB, Nair A, Jin S, et al. Editorial: Perspectives from the front lines of COVID-19: New York City ophthalmology resident narratives. Curr Opin Ophthalmol 2020;31(05):386-388

8 Tran EM, Scott IU, Clark MA, Greenberg PB. Assessing and promoting the wellness of United States Ophthalmology Residents: a survey of program directors. J Surg Educ 2018;75(01):95-103

9 Hu Y-Y, Ellis RJ, Hewitt DB, et al. Discrimination, abuse, harassment, and burnout in surgical residency training. N Engl J Med 2019;381(18):1741-1752

10 Alotaibi AK, Alsalim A, Alruwaili F, et al. Burnout during ophthalmology residency training: a national survey in Saudi Arabia. Saudi J Ophthalmol 2019;33(02):130-134

11 Shanafelt TD, Noseworthy JH. Executive leadership and physician well-being: nine organizational strategies to promote engagement and reduce burnout. Mayo Clin Proc 2017;92(01):129-146

12 Shanafelt T, Goh J, Sinsky C. The business case for investing in physician well-being. JAMA Intern Med 2017;177(12): 1826-1832

13 Shanafelt TD, Bradley KA, Wipf JE, Back AL. Burnout and selfreported patient care in an internal medicine residency program. Ann Intern Med 2002;136(05):358-367

14 West CP, Huschka MM, Novotny PJ, et al. Association of perceived medical errors with resident distress and empathy: a prospective longitudinal study. JAMA 2006;296(09):1071-1078

15 Firth-Cozens J, Greenhalgh J. Doctors' perceptions of the links between stress and lowered clinical care. Soc Sci Med 1997;44 (07):1017-1022

16 Shanafelt TD, Balch CM, Bechamps G, et al. Burnout and medical errors among American surgeons. Ann Surg 2010;251(06): 995-1000

17 Venincasa MJ, Cai LZ, Gedde SJ, Uhler T, Sridhar J. Current applicant perceptions of the ophthalmology residency match. JAMA Ophthalmol 2020;138(05):460-466

18 Review Committee for Ophthalmology. Required Minimum Number of Procedures for Graduating Residents in Ophthalmology. Accessed November 9, 2020 at: https://www.acgme.org/Portals/ 0/PFAssets/ProgramResources/240_Oph_Minimum_Numbers. pdf?ver $=2015-11-06-120652-043$

19 Coronavirus: Why are infections rising again in US? - BBC news. Accessed November 9, 2020 at: https://www.bbc.com/news/election-us-2020-54423928

20 Vargo E, Ali M, Henry F, et al. Cleveland Clinic Akron General Urology Residency Program's COVID-19 experience. Urology 2020;140:1-3

21 Rosen GH, Murray KS, Greene KL, Pruthi RS, Richstone L, Mirza M. Effect of COVID-19 on urology residency training: a nationwide survey of Program Directors by the Society of Academic Urologists. J Urol 2020;204(05):1039-1045

22 Amparore D, Claps F, Cacciamani GE, et al. Impact of the COVID-19 pandemic on urology residency training in Italy. Minerva Urol Nefrol 2020;72(04):505-509

23 Crosby DL, Sharma A. Insights on Otolaryngology Residency Training during the COVID-19 pandemic. Otolaryngol Head Neck Surg 2020;163(01):38-41 
24 Zingaretti N, Contessi Negrini F, Tel A, Tresoldi MM, Bresadola V, Parodi PC. The impact of COVID-19 on plastic surgery residency training. Aesthetic Plast Surg 2020;44(04):1381-1385

25 Bitonti G, Palumbo AR, Gallo C, et al. Being an obstetrics and gynaecology resident during the COVID-19: Impact of the pandemic on the residency training program. Eur J Obstet Gynecol Reprod Biol 2020;253:48-51

26 Rothrock RJ, Maragkos GA, Schupper AJ, et al. By the numbers analysis of effect of COVID-19 on a neurosurgical residency at the epicenter. World Neurosurg 2020;142:e434-e439

27 Huntley RE, Ludwig DC, Dillon JK. Early effects of COVID-19 on oral and maxillofacial surgery residency training-results from a national survey. J Oral Maxillofac Surg 2020;78(08):1257-1267

28 Alvarez H, Rodesch G, Garcia-Monaco R, Lasjaunias P. Embolisation of the ophthalmic artery branches distal to its visual supply. Surg Radiol Anat 1990;12(04):293-297

29 Pertile D, Gallo G, Barra F, et al; SPIGC Working Group. The impact of COVID-19 pandemic on surgical residency programmes in Italy: a nationwide analysis on behalf of the Italian Polyspecialistic Young Surgeons Society (SPIGC). Updates Surg 2020;72(02): 269-280

30 Odedra D, Chahal BS, Patlas MN. Impact of COVID-19 on Canadian Radiology Residency Training Programs. Can Assoc Radiol J 2020; 71(04):482-489

31 Mishra D, Nair AG, Gandhi RA, et al. The impact of COVID-19 related lockdown on ophthalmology training programs in India-outcomes of a survey. Indian J Ophthalmol 2020;68(06):999-1004

32 Silva N, Laiginhas R, Meireles A, Barbosa Breda J. Impact of the COVID-19 pandemic on ophthalmology residency training in Portugal. Acta Med Port 2020;33(10):640-648

33 Ferrara M, Romano V, Steel DH, et al; OphthaTraining Group. Reshaping ophthalmology training after COVID-19 pandemic. Eye (Lond) 2020;34(11):2089-2097

34 Nayak B, Sen S, Parija S. Effect of COVID-19 on ocular diseases and ophthalmology residency training program-a developing country's perspective. Indian J Ophthalmol 2020;68(07):1491

35 Pasricha ND, Haq Z, Ahmad TR, et al. Remote corneal suturing wet lab: microsurgical education during the COVID-19 pandemic. J Cataract Refract Surg 2020;46(12):1667-1673

36 Wagner RS. Strabismus surgical training during the COVID-19 pandemic. J Pediatr Ophthalmol Strabismus 2020;57(04): 211-212

37 DeMaria LN, Tran AQ Tooley AA, Lisman RD, Belinsky I, Kim ET. Oculoplastics education in the coronavirus 2019 pandemic with virtual suturing curriculum. Ophthal Plast Reconstr Surg 2020;36 (05):515-516

38 Ramirez DA, Dawoud SA. Resident perspectives on COVID-19: three takeaways. Am J Ophthalmol 2020;220:A2-A4

39 Rogers E, Polonijo AN, Carpiano RM. Getting by with a little help from friends and colleagues: testing how residents' social support networks affect loneliness and burnout. Can Fam Physician 2016; 62(11):e677-e683
40 Khanna RC, Honavar SG, Metla AL, Bhattacharya A, Maulik PK. Psychological impact of COVID-19 on ophthalmologists-in-training and practising ophthalmologists in India. Indian J Ophthalmol 2020;68(06):994-998

41 Almater AI, Tobaigy MF, Younis AS, Alaqeel MK, Abouammoh MA. Effect of 2019 coronavirus pandemic on ophthalmologists practicing in Saudi Arabia: a psychological health assessment. Middle East Afr J Ophthalmol 2020;27(02):79-85

42 Ting DSW, Lin H, Ruamviboonsuk P, Wong TY, Sim DA. Artificial intelligence, the internet of things, and virtual clinics: ophthalmology at the digital translation forefront. Lancet Digit Health 2020;2(01):e8-e9

43 Ting DSW, Carin L, Dzau V, Wong TY. Digital technology and COVID-19. Nat Med 2020;26(04):459-461

44 Berkenstock MK, Liberman P, McDonnell PJ, Chaon BC. Changes in patient visits and diagnoses in a large academic center during the COVID-19 pandemic. BMC Ophthalmol 2021;21(01):139

45 Hu K, Patel J, Swiston C, Patel BC. Ophthalmic manifestations of coronavirus (COVID-19). StatPearls Publishing2021. Accessed June 24, 2021 at: http://www.ncbi.nlm.nih.gov/books/NBK556093/

46 Mehta S, Pandey A. Rhino-orbital mucormycosis associated with COVID-19. Cureus 2020;12(09):e10726

47 Aggarwal K, Agarwal A, Jaiswal N, et al. Ocular surface manifestations of coronavirus disease 2019 (COVID-19): a systematic review and meta-analysis. PLoS One 2020;15(11):e0241661

48 Stevens DV, Tran AQ Kim E. Complications of orbital emphysema in a COVID-19 patient. Ophthalmology 2020;127(07):990

49 Sengillo JD, Kunkler AL, Medert C, et al. UV-photokeratitis associated with germicidal lamps purchased during the COVID-19 pandemic. Ocul Immunol Inflamm 2021;29(01):76-80

50 Hollick EJ, Allan BD. Live surgery: national survey of United Kingdom ophthalmologists. J Cataract Refract Surg 2008;34 (06):1029-1032

51 van Gog T, Paas F, Marcus N, Ayres P, Sweller J. The mirror neuron system and observational learning: implications for the effectiveness of dynamic visualizations. Educ Psychol Rev 2008;21(01): 21-30

52 Staropoli PC, Gregori NZ, Junk AK, et al. Surgical simulation training reduces intraoperative cataract surgery complications among residents. Simul Healthc 2018;13(01):11-15

53 Belyea DA, Brown SE, Rajjoub LZ. Influence of surgery simulator training on ophthalmology resident phacoemulsification performance. J Cataract Refract Surg 2011;37(10):1756-1761

54 Sutherland LM, Middleton PF, Anthony A, et al. Surgical simulation: a systematic review. Ann Surg 2006;243(03):291-300

55 Thomsen ASS, Subhi Y, Kiilgaard JF, la Cour M, Konge L. Update on simulation-based surgical training and assessment in ophthalmology: a systematic review. Ophthalmology 2015;122(06): 1111-1130.e1

56 Dawe SR, Pena GN, Windsor JA, et al. Systematic review of skills transfer after surgical simulation-based training. Br J Surg 2014; 101(09):1063-1076 